\section{The association between activi- ty levels and skin moisturising function in adults}

\author{
Oizumi Ryosuke, ${ }^{1}$ Sugimoto Yoshie, ${ }^{2}$ \\ Aibara Hiromi \\ ${ }^{1}$ School of Nursing Doctoral Course, and \\ ${ }^{2}$ School of Nursing, Osaka Prefecture \\ University Graduate Habikino, habiki- \\ no-shi, Osaka, Japan
}

\begin{abstract}
Factors associated with skin moisture retention include age and lifestyle, such as diet and sleep efficiency. However, the impact of exercise habits on skin moisturising function is unclear. We surveyed 86 participants from a Japanese university about their activity levels using the International Physical Activity Questionnaire. We also examined their skin moisturising function, measuring stratum corneum (SC) hydration levels and transepidermal water loss (TEWL). Comparisons of participants' activity levels, SC hydration, and TEWL, accounting for their gender and age differences, revealed activity levels significantly related to differences in SC hydration levels. Results of multiple comparisons showed increased activity relates to significantly higher SC hydration - the higher the activity levels, the higher the hydration. No difference was found in TEWL. The results suggest exercise habits can prevent dry skin. The findings may be useful for the prevention and treatment of dry skin and promoting the benefits of exercise.
\end{abstract}

\section{Introduction}

Skin problems can develop regardless of age, and skin problems can range from acne in young people to dryness in the elderly. These conditions may cause mental anxiety and physical issues such as itchiness, thereby negatively affecting one's quality of life (QoL). ${ }^{1,2}$ Keeping the skin in good condition is important for both physical and mental health. It is, therefore, important to maintain proper skin conditioning.

Important functions of the skin include moisture retention, which regulates the release of water from the inside of the body to the atmosphere, and the barrier function, preventing chemicals and microbes from the environment from entering the body. ${ }^{3}$ The stratum corneum is important to both the moisturising and barrier functions. ${ }^{4}$ Skin problems are caused by disruption of the skin barrier function ${ }^{3}$ moisturising and barrier function are strongly related. ${ }^{4}$ TransEpidermal Water Loss (TEWL) and stratum corneum (SC) hydration are useful for measuring the skins' moisturising and barrier functions. ${ }^{3,5}$ In previous studies, researchers have examined these indicators and have identified several factors that affect these functions (e.g. daily skincare habits, bathing habits, smoking, rest, diet, etc.). ${ }^{6-13}$

However, the association between skin moisturising and barrier function and exercise is not well understood. Known effects of exercise on the skin are blood flow motion increase ${ }^{14}$ and changes in skin temperature. ${ }^{15}$ It is also known that SC hydration increases temporarily immediately following a single high-intensity exercise session but significantly decreases after 120 minutes to a level lower than before the bout of exercise. ${ }^{16}$ On the other hand, compared to occasional, single sessions of exercise, a long-term endurance exercise habit yields improvements to the exerciser's skin structure, such as to the stratum corneum thickness. ${ }^{17,18}$ The decrease in SC hydration after exercise is caused by sweating, which causes the stratum corneum to swell, increasing the outflow of hydro-soluble natural moisturising factors. ${ }^{16}$ This effect of SC expansion on SC hydration is transient. ${ }^{16}$ The short-term and long-term effects of exercise on the skin moisturising function need to be interpreted separately. It is not clear whether the impacts on the skin differ according to the term of endurance exercise or the intensity of endurance exercise.

Therefore, it is necessary to examine the effects of medium- to long-term exercise habits on the skin moisturising and barrier functions. This study examined the relationship between exercise habits and skin moisture retention and barrier function in the last month. If the relationship between the moisturising function of the skin and the level of exercise is clarified, a new option for exercise instruction will emerge as a method for preventing skin trouble in clinical practice.

\section{Materials and Methods}

\section{Study design}

This cross-sectional, observational study was conducted in Japan from July to October 2019.

\section{Participants}

We recruited students and staff from $X$ University to ensure the participant group represented a wide range of ages. However,
Correspondence: Oizumi Ryosuke, Osaka Prefecture University Graduate School of nursing doctoral course, Habikino, habikinoshi, 3-7-30, Osaka, 583-8555, Japan.

Tel.: +81.728132654.

E-mail: mb601009@edu.osakafu-u.ac.jp

Key words: lifestyle, exercise, skin physiological phenomena, skin ageing.

Acknowledgements: We greatly appreciate the cooperation of the participants and staff members in this study.

Contributions: RO, YS, and HA conceived and designed the study. RO contributed to obtaining and analysing the data. RO, YS, and HA drafted and revised the manuscript. All the authors critically reviewed and approved the submitted version of the manuscript.

Conflict of interest: The authors declare no conflict of interest.

Funding: None.

Ethics approval and consent to participate: This study was conducted after gaining permission from the research ethics committee of University Graduate School of Nursing (Approval No. 2019-29). Consent was obtained before submission.

Please cite this article as: Ryosuke $O$, Yoshie $S$, Hiromi $A$. The association between activity levels and skin moisturising function in adults. Dermatol Rep 2021;13:8811.

Received for publication: 25 July 2020.

Accepted for publication: 11 December 2020.

This work is licensed under a Creative Commons Attribution-NonCommercial 4.0 International License (CC BY-NC 4.0).

(C) Copyright: the Author(s), 2021

Licensee PAGEPress, Italy

Dermatology Reports 2021; 13:8811

doi:10.4081/dr.2021.8811

those with chronic skin diseases such as atopic dermatitis were excluded. Thus, a total of 86 participants without skin disorders were thus recruited. This study was conducted as part of a health check-up program to modify lifestyle habits. All participation was voluntarily.

\section{Data collection}

This study analysed two skin moisturising function parameters based on data from self-administered questionnaires concerning participant exercise habits. We confirmed that no responses had been omitted when collecting the questionnaires. All participants completed these in a room set to a 
temperature of $20-22 \square$, with humidity levels measuring between 40-60\%. Skin moisturising function parameters were measured after 20 minutes of rest in the room where the questionnaire was answered.

The self-administered questionnaire included questions regarding sex, age, and exercise habits. Exercise habits were measured using the Japanese version of the International Physical Activity Questionnaire-short version (IPAQ-SF). ${ }^{19}$ Reliability and validity were previously confirmed. ${ }^{20}$ The intensity of exercise was divided into three activity levels according to the responses to IPAQ. The questionnaire was analysed according to the guidelines. ${ }^{21}$

Skin moisturising functions were evaluated based on TEWL and SC hydration. TEWL measurements were taken from the centre point of each participant's right forearm $(8 \mathrm{~cm}$ point on the palm side from the elbow fossa centre). This method was used due to the ease of obtaining stable values. ${ }^{8}$ All measurements were performed for 20 seconds; the average value from the last 10 seconds was used as data. The Tewameter TM300 was used for this purpose (Courage + Khazaka electronic GmbH, Köln, Germany).

To influence the measurement index, ${ }^{8}$ we requested that participants adhere to the following three rules:

i. Do not apply body cream or similar substances to the measurement site for at least 12 hours prior to the measurement.

ii. Do not consume caffeinated beverages (e.g., coffee or tea) or smoke for at least three hours prior to the study. iii. Do not engage in strenuous exercise for at least one hour prior to the study.

\section{Ethical consideration}

This study was conducted after gaining permission from the research ethics committee of University Graduate School of Nursing (Approval No. 2019-29). Participants were informed that they had the right to decline participation and could withdraw from the study at any time. Participation was completely voluntary, and all participants received both oral and written information about the study purpose, contents, and extent. They were assured that all responses were confidential. Specifically, participant confidentiality was protected by giving each participant a unique code number prior to data collection and analysis. Also, collected questionnaires were kept in a locked cabinet. Consent checkboxes were included on each questionnaire form; participant consent was indicated by checking the respective boxes before submitting the forms.

\section{Data analysis}

All data were calculated by a web application named langtest.jp at http://langtest.jp (accessed July 1, 2020).22 Descriptive statistics were presented as means and standard deviations (SD) for continuous variables, while numbers (\%) were used for categorical variables.

Differences between activity level (Low, Moderate, or High) and skin moisturising function were examined using Kruskal-Wallis test. Pairwise comparisons were completed using the Mann-Whitney U test and the Holm adjustment to control for Type I errors across tests. We deemed $p$-values less than 0.05 to be significant.

\section{Results}

Table 1 shows participants' characteristics and skin function, and Kruskal-Wallis test results. Data from all 86 participants were included in the analyses. Many of the participants were women $(83.7 \%)$. The median (Min, Max) age was $30.0(20,62)$ years. There was no difference in age between activity levels $(\mathrm{p}=0.92)$ and no difference in TEWL $(p=0.22)$. However, there was a statistically significant between activity level in SC $(\mathrm{p}<0.001)$.

To the difference of each group gender ratio were large, confirmed that there was no difference in the skin moisturising function between the genders (Table 2). Since there was a significant difference in SC between activity levels, a pairwise comparison was performed. Figure 1 shows the distribution of SC in each group. All pairwise comparisons were statistically significant (Table 3). The higher the activity level, the higher the SC. The Effect size [95\% CI] was large between Low and High $(\mathrm{r}=0.52[0.26$, $0.70]$ ).

\section{Discussion}

We investigated the association between activity level and skin-moisturising function using a self-administered ques-

Table 1. Characteristics and skin function of participant.

\begin{tabular}{|c|c|c|c|c|c|}
\hline $\begin{array}{l}\text { Characteristics and skin function } \\
(\mathrm{n}=86)\end{array}$ & $\begin{array}{c}\text { Total sample } \\
(\mathrm{n}=34)\end{array}$ & $\begin{array}{l}\text { Low level } \\
(\mathrm{n}=42)\end{array}$ & $\begin{array}{l}\text { Moderate level } \\
\quad(n=10)\end{array}$ & High level & P-value \\
\hline Age, median (min, max) & $30.0(20,62)$ & $33.0(20,57)$ & $29.5(20,62)$ & $28.0(21,56)$ & 0.92 \\
\hline $\begin{array}{c}\text { Gender, n (\%) } \\
\text { Male } \\
\text { Female }\end{array}$ & $\begin{array}{l}14(16.3) \\
72(83.7)\end{array}$ & $\begin{array}{c}3(8.8) \\
31(91.2)\end{array}$ & $\begin{array}{c}6(14.3) \\
36(85.7)\end{array}$ & $\begin{array}{l}5(50.0) \\
5(50.0)\end{array}$ & \\
\hline SC, median (min, max) & $38.5(22.7,56.1)$ & $34.4(24.3,48.8)$ & $39.9(22.7,53.4)$ & $46.5(35.1,56.1)$ & $<0.001$ \\
\hline TEWL $\left(\mathrm{g} / \mathrm{h} / \mathrm{m}^{2}\right)$, median (min, max) & $4.96(2.42,8.95)$ & $4.86(2.42, .95)$ & $4.80(2.56,7.13)$ & $6.06(2.71,8.43)$ & 0.22 \\
\hline
\end{tabular}

Kruskal-Wallis test.

Table. 2. SC and TEWL between genders.

\begin{tabular}{lcccc} 
& Male $(\mathrm{n}=14)$ & Female $(\mathrm{n}=7 \mathbf{2})$ & Z-value & P-value \\
SC, median (min, max) & $40.5(27.8,53.4)$ & $38.25(22.7,56.1)$ & -1.176 & .240 \\
TEWL $\left(\mathrm{g} / \mathrm{h} / \mathrm{m}^{2}\right)$, median (min, max) & $4.62(2.42,8.35)$ & $4.99(2.56,8.95)$ & -1.234 & .217 \\
\hline
\end{tabular}


Table 3. Comparison of SC between each group.

\begin{tabular}{lccc} 
& Z-value & P-value & Effect sive (r) [95\% Cl] \\
Low-Moderate & -2.722 & 0.01 & $0.31[0.09,0.50]$ \\
Low-High & -3.417 & $<0.01$ & $0.52[0.26,0.70]$ \\
\hline Moderate-High & -2.415 & 0.02 & $0.34[0.07,0.56]$ \\
\hline
\end{tabular}

Mann-Whitney's U test.

tionnaire about exercise habits and by examining two kinds of skin moisturising and barrier function. The results suggest that active people have moist skin.

In previous studies, long-term endurance exercises habits have been shown to improve skin structure, ${ }^{17,18}$ but, after a single endurance exercise, SC hydration is reduced. ${ }^{16}$ However, these studies did not clarify the association between exercise habits and skin's moisturising function. An important finding of this study is that exercise habits may improve skin moisturising function and that higher-intensity exercise may promote the skin-moisturising function.

This study results showed the moderateand high-activity level habit groups have high skin moister levels than the low-activity level habit group. This result suggests that exercise habits may improve skin moisturising function. Declines in skin functions, such as skin moisturising and barrier function, are thought to be driven by pronounced mitochondrial DNA deletions. ${ }^{23}$ Therefore, it is necessary to stimulate mitochondrial biosynthesis to improve skin function. Endurance exercise induces IL-5, and IL-5 promotes biosynthesis mitochondria; thus, skin construction improves. ${ }^{18}$ Skin construction is associated with SC hydration. ${ }^{4}$ The moistened skin of active individuals may be due to improved skin structure due to mitochondrial biosynthesis. Exercise habits can improve the structure of the skin and improve the skin's moisturising function. However, it is not clear what type of exercise is most effective for this purpose. The IPAQ used in this study does not provide specific exercise details. Therefore, it is not clear whether the participants' exercise was endurance exercise or not. Possible differences in the effects of different types of exercise, such as endurance training compared to strength training, on skin moisturising function should be investigated. Additionally, results from previous studies have suggested that diet and sleep efficiency also affect skin moisturising function. ${ }^{12,13}$ Thus, further studies with adjustments for factors such as dietary habits and sleep are needed, as exercise may also improve sleep conditions. $^{24}$

One of the factors in the lack of relationship between activity level and TEWL

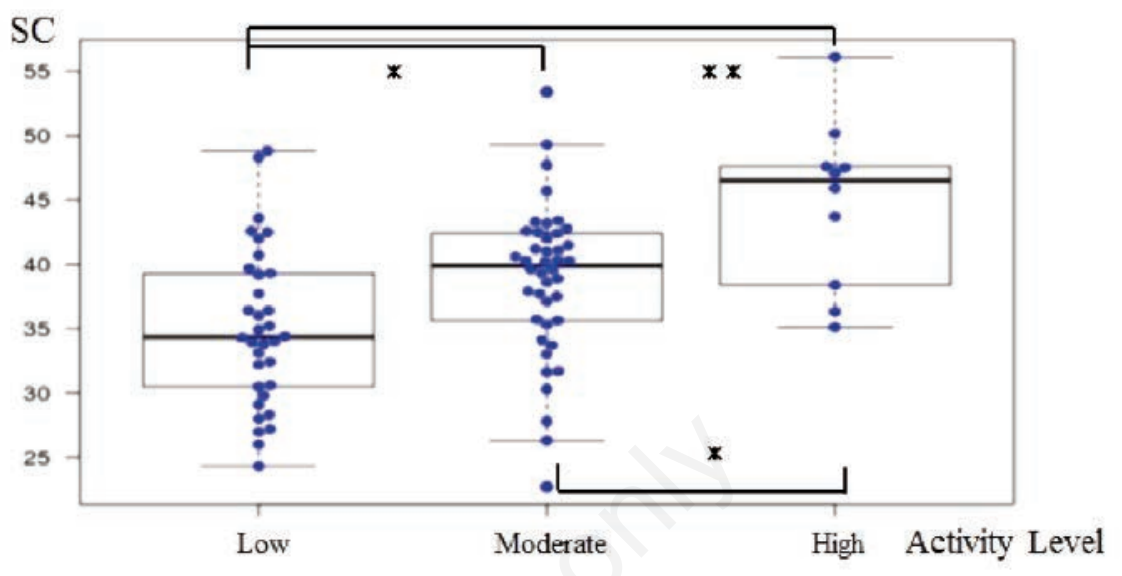

Figure 1. Distribution of SC in each group. ${ }^{*}<0.05$ and ${ }^{* *}<0.01$.

may be that participants in this study were not older, and not had skin diseases. TEWL depends on physical structures such as the lamellar bodies of the stratum corneum, ${ }^{25}$ the structure of which is deteriorated by chronic dermatitis and ageing. ${ }^{26,27}$ Therefore, the healthy adults who participated in this study had healthy stratum corneum structure with no factors that could worsen its structure. Exercise improves the structure of the stratum corneum in older adults, ${ }^{18}$ but this may only be effective for those with originally deteriorated stratum corneum structure. Future studies should be conducted in the elderly and people with skin diseases to clarify the relationship between TEWL and activity levels.

The results of this study suggest that exercise may increase SC hydration. SC hydration has an association with skin barrier function. ${ }^{4}$ Therefore, as exercise raises $\mathrm{SC}$ hydration, exercise may prevent skin problems. Hence, studies should also be conducted to determine if the increases SC hydration resulting from exercise can prevent the development of skin problems.

There are several limitations to the present study. First, cross-sectional studies cannot prove causality. Therefore, further investigation by a prospective study is necessary. Second, our study participants were limited to people belonging to one university, and the group was mostly comprised of women. Thus, studies with diverse participant groups are necessary. Despite these limitations, this study results provide evidence of an association between activity level and SC hydration. Further research will help to raise awareness of the exercise to dermatology patients and people living in the community.

\section{Conclusions}

People with higher activity levels are likely to have higher SC hydration than those with lower activity levels - the higher the activity level, the higher the SC hydration. Exercise improves the skin's ability to retain moisture and may prevent future skin problem. Further studies into this relationship may help in the treatment of dry skin and related skin problems.

These results, along with those from future studies with diverse participants, may assist dermatologists and primary care doctors in advising their patients about skin problems and hydration. They may also be used in public service announcements and health-related promotional materials describing the benefits of regular exercise.

\section{References}

1. Paul C, Maumus-Robert S, MazereeuwHautier J, et al. Prevalence and risk factors for xerosis in the elderly: a crosssectional epidemiological study in pri- 
mary care. Dermatology 2011;223:2605.

2. Callender VD, Alexis AF, Daniels SR, et al. Racial differences in clinical characteristics, perceptions and behaviors, and psychosocial impact of adult female acne. J Clin Aesthet Dermatol 2014;7:19-31.

3. Proksch E, Brandner JM, Jensen JM. The skin: an indispensable barrier. Exp Dermatol 2008;17(12):1063-72.

4. Madison KC. Barrier function of the skin: "la raison d "être" of the epidermis. J Invest Dermatol 2003;121:23141.

5. Berardesca E, Loden M, Serup J, et al. The revised EEMCO guidance for the in vivo measurement of water in the skin. Skin Res Technol 2018;24:351-8.

6. Fujino Y, Yasuda T, Douken Y, et al. Skin physiological function and skin care in community-dwelling elders. J Nurs Soc University of Toyama 2016;15:93-104.

7. Iizaka S. Skin hydration and lifestylerelated factors in community-dwelling older people. Arch Gerontol Geriatr 2017;72:121-6.

8. Negoro S, Hayama Y, Inoue T. The Dry Situation of Community-dwelling Elderly Woman's Skin, and the Actual Condition of the Lifestyle Relevant to Dryness. Jpn Med Assoc J 2013;21:237-43.

9. Asakura K, Nishiwaki Y, Milojevic A, et al. Lifestyle factors and visible skin aging in a population of Japanese elders. J Epidemiol 2009;19:251-9.

10. Plessis JD, Stefaniak A, Eloff F, et al. International guidelines for the in vivo assessment of skin properties in nonclinical settings: Part 2. trans epidermal water loss and skin hydration. Skin Res
Technol 2013;19:265-78.

11. Altemus M, Rao B, Dhabhar FS, et al. Stress-induced changes in skin barrier function in healthy women. J Invest Dermatol 2001;117:309-17.

12. Draelos ZD. Aging skin: the role of diet: facts and controversies. Clin Dermatol 2013;31:701-6.

13. Yoshizaki T, Kimira Y, Mano H, et al. Association between Skin Condition and Sleep Efficiency in Japanese Young Adults. J Nutr Sci Vitaminol 2017;63:15-20.

14. Rossi M, Santoro G, Maurizio S, Carpi A. Spectral analysis of skin blood flowmotion before and after exercise in healthy trained and in sedentary subjects. Int J Sports Med 2006;27:540-5.

15. Neves EB, Vilaca-Alves J, Antunes N, et al. Different responses of the skin temperature to physical exercise: Systematic review. Conf Proc IEEE Eng Med Biol Soc 2015;1307-10.

16. Eda N, Shimizu K, Suzuki S, et al. Effects of High-Intensity Endurance Exercise on Epidermal Barriers against Microbial Invasion. J Sports Sci Med 2013; 12:44-51.

17. Safdar A, Bourgeois JM, Ogborn DI, et al. Endurance exercise rescues progeroid aging and induces systemic mitochondrial rejuvenation in mtDNA mutator mice. Proc Natl Acad Sci USA 2011;108:4135-40.

18. Crane JD, MacNeil LG, Lally JS, et al. Exercise-stimulated interleukin-15 is controlled by AMPK and regulates skin metabolism and aging. Aging Cell 2015;14;625-34.

19. Murase N., Katsumura T., Ueda C, et al. Validity and reliability of Japanese version of International Physical Activity Questionnaire. J Health Welfare Stat
2002;49:1-9.

20. Craig CL, Marshall AL, Sjöström M, et al. International physical activity questionnaire: 12-country reliability and validity. Med Sci Sports Exerc 2003;35:1381-95.

21. Guidelines for Data Processing and Analysis of the International Physical Activity Questionnaire (IPAQ). https://sites.google.com/site/theipaq/sc oring-protocol. (accessed July 1, 2020)

22. Mizumoto A, Plonsky L. R as a Lingua Franca: Advantages of Using $\mathrm{R}$ for Quantitative Research in Applied Linguistics. Applied Linguistics 2016;37:284-91.

23. Lu CY, Lee HC, Fahn HJ, Wei YH. Oxidative damage elicited by imbalance of free radical scavenging enzymes is associated with large-scale mtDNA deletions in aging human skin. Mutat Res 1999;423:11-21.

24. Kim K, Uchiyama M, Okawa M, et al. An epidemiological study of insomnia among the Japanese general population. Sleep 2000;23:4-7.

25. Elias PM. Stratum corneum defensive functions: an integrated view. J Invest Dermatol 2005;125:183-200.

26. Berardesca E, Fideli D, Borroni G, et al. In vivo hydration and water-retention capacity of stratum corneum in clinically uninvolved skin in atopic and psoriatic patients. Acta Derm Venereol 1990;70:400-4.

27. Leveque JL, Corcuff P, de Rigal J, et al. In vivo studies of the evolution of physical properties of the human skin with age. Int J Dermatol 1984;23:322-9. 